\title{
Configuration Interaction Singles with Spin-Orbit Coupling: Constructing Spin-Adiabatic States and Their Analytical Nuclear Gradients
}

\author{
Nicole Bellonzi, Gregory Medders, Evgeny Epifanovsky and Joseph E. Subotnik
}

(Dated: June 21, 2018)

\begin{abstract}
For future use in modeling photoexcited dynamics and intersystem crossing, we calculate spin-adiabatic states and their analytical nuclear gradients within CIS theory. These energies and forces should be immediately useful for surface hopping dynamics, which are natural within an adiabatic framework. The resulting code has been implemented within the Q-Chem software and preliminary results suggest that the additional cost of including SOC within the singles-singles block is not large.
\end{abstract}

\section{INTRODUCTION}

Intersystem Crossing (ISC) is a key relaxation pathway for many photo-excited systems. For instance, several aromatic ketones and aldehydes are known to undergo ISC phosphorescence with almost unity quantum efficiency [1]. As another example, organometallics with heavy elements (e.g. platinum) are also known to undergo ISC very efficiently[2]. Moreover, because of the long lifetimes of triplet states, recent work in photocatalyzed organic synthesis has sought to isolate organic precursors with propensities to form excited state triplets, so as to maximize yields of photocatalyzed product [3].

Now, in general, it is well known that triplets tend to be the lowest energy excited states due to antisymmetry and exchange [4]. Thus, ISC is almost always thermodynamically accessible. Nevertheless, many molecules do not undergo ISC, highlighting that whether or not a triplet forms after photoexcitation is dictated $\boldsymbol{b} \boldsymbol{y}$ dynamics and not by electronic structure. Thus, to make predictions about triplet formation, we must run dynamics simulations. And, if we are to run nonadiabatic dynamics, the key question to ask is: what is the coupling between the singlet and triplet states? There are many such operators for only singlet to triplet intersystem crossing, including spin-orbit coupling (SOC), hyperfine couplings, and spin-spin couplings. In the present paper, we will focus on ISC as induced 
by SOC, for which El-Sayed's rule is applicable[5]. A few words are now appropriate about the exact form of the SOC operator.

Formally, the SOC operator is derived a consequence of the Dirac equation and cannot be derived with a non-relativistic theory of quantum mechanics. Nevertheless, up to a factor of two [6], the form for the SOC operator can be rationalized with straightforward classical electromagnetic arguments, and nowadays, it is standard within the quantum chemistry community to use the so-called Breit-Pauli perturbative form of the SOC operator within nonrelativistic quantum mechanics [7. According to the Breit-Pauli form, the SOC is a vector operator with one and two electron components. The one electron component is:

$$
\mathbf{V}_{\mathbf{S O}}=-\frac{\alpha_{0}^{2}}{2} \sum_{j, A} \frac{Z_{A}}{\left|\mathbf{r}_{j A}\right|^{3}}\left(\mathbf{r}_{j A} \times \mathbf{p}_{j}\right) \cdot \mathbf{s}_{j}
$$

where $\alpha_{0}$ is the fine structure constant, and $j$ and $A$ index the electrons and nuclei, respectively. $Z_{A}$ is the charge of nucleus $A, \mathbf{s}_{j}$ is the spin operator of the $j$ electron, $\mathbf{r}_{j A}$ is the distance between electron $j$ and nucleus $A$, and $\mathbf{p}_{j}$ is the momentum of electron $j$. In this paper, we will restrict ourselves to the one-electron piece of the SOC operator; others have shown that a screened one electron SOC term can capture many of the effects of the total SOC operator [8].

Now, given an operator for the SOC, suppose we would like to run Tully's Fewest Switches Surface Hopping (FSSH) [ to determine ISC rates and branching ratios. This problem has been considered by several authors in recent years [10 12. For the reader not familiar with Tully's algorithm, a few words about FSSH are now appropriate. The basic input to an FSSH trajectory are 1. adiabatic potential energies surfaces, 2. nuclear gradients, and 3. derivative couplings, and the basic ansatz of FSSH is to run dynamics on adiabatic surfaces, while hopping between surfaces to account for electronic relaxation. One key element of surface hopping dynamics is that all dynamics are propagated along adiabatic surfaces. This choice of surface ensures that barrier crossings (without tunneling) are correct and also that detailed balance is preserved approximately $[9,13,14]$. This choice furthermore gives us the correct choice of hopping direction - the derivative coupling [15 18]. In fact, the FSSH algorithm can be justified approximately only when the dynamics are run along an adiabatic basis [19]. Even when the coupling is small, an adiabatic basis is still feasible [20 22]. And finally, recent work by several authors has shown that (when studying ISC), the dynamics can have large errors if one propagates along spin-diabats (i.e. singlet or triplet surfaces) 
rather than spin-adiabats (i.e. surfaces that mix spins) [10, 11, 19, 23].

Altogether, the evidence above suggests that, in order to run ISC dynamics with FSSH, we should construct spin-adiabatic wavefunctions, i.e. the eigenstates of $\mathbf{H}_{\mathrm{el}}+\mathbf{V}_{\mathrm{SO}}$ (see Eqn. 18 below) 24]. At this point, however, we must remind ourselves that the cost of diagonalizing the supermatrix will be large. And in fact, we must also recall that mixing one singlet and one triplet does not result in a system with two electronic states; instead, it results in a system with four electronic states because the triplets are always triply degenerate; of course all degeneracy is broken by SOC. Moreover, it would make no sense to include only one of the three triplets (say $m_{s}=0$ triplet) because then the calculation would depend spuriously on the artificial choice of lab frame and molecular orientation. And because the phase of a coupling can be essential (e.g. near a conical intersection), it is not reasonable to reduce the system to a single triplet with, e.g. $\left|\mathbf{V}_{\text {So }}\right|^{2}$, as an average (rotationally invariant) perturbative matrix element. Instead, for a rotationally invariant calculation, one must include all of the components of the triplet and diagonalize the full Hamiltonian (which also includes all of the vector components of the SOC operator, see Eqn. 14). And yet, given that the most expensive piece of a CIS calculation is multiplication of the trial amplitudes by the two electron integrals, and given the fact that the two electron integrals do not mix spin symmetry, it should be possible to compute spin-adiabat electronic states, as well as spin-adiabatic gradients, with minimal cost 25].

With all of this background in mind, our goal for this paper is to derive and implement an algorithm for quickly generating spin-adiabatic states and their gradients within the context of configuration interaction singles (CIS), a popular and computationally efficient method to generate excited spin-diabatic state energies and amplitudes. Similar previous work with a semiempirical approach was performed by Granucci and Persico [26].

\section{THEORETICAL METHODS}

\section{A. Notation}

Establishing correct notation will be essential for our problem with spatial and spin degrees of freedom. Henceforward, lowercase Greek letters $\mu \nu \lambda \gamma \omega$ index atomic orbitals. Lowercase Roman letters pqrs index general molecular orbitals from the Hartree Fock ground 
state $\left(|p\rangle=\sum_{\mu} C_{\mu p}|\mu\rangle\right)$; abcd index specifically virtual orbitals, and $i j k l m$ index specifically occupied orbitals. Spin orbitals are represented by bold type as $\mathbf{p}$ or $\boldsymbol{\mu}$, or when explicitness is required, with subscripts as follows: $p_{\alpha}$ for up spin, $p_{\beta}$ for down spin, or $p_{\sigma}$ for either spin. A single excited state is defined by $\left|\Phi_{\mathbf{i}}^{\mathbf{a}}\right\rangle \equiv a_{\mathbf{a}}^{\dagger} a_{\mathbf{i}}\left|\Phi_{\mathrm{HF}}\right\rangle$. The singlet spin-diabat is indexed by $(s)$ and triplet spin-diabats $(t)$ are indexed by $m_{s}=-1,0,+1$. The four spin-diabats (one singlet and three triplets) can be indexed by $\epsilon \in\left\{s, m_{s}\right\}$. Finally, note that some quantities below will be complex; an asterisk $(*)$ will denote the complex conjugate.

\section{B. Standard CIS}

We begin by outlining standard configuration interaction singles (CIS) theory of excited states. In this work, we use the closed-shell restricted form of the CIS equations. The standard CIS algorithm calculates the eigenstates of the electronic Hamiltonian,

$$
\begin{aligned}
\mathbf{H}_{\mathrm{el}} & =\sum_{\mathbf{p q}} h_{\mathbf{p q}} a_{\mathbf{p}}^{\dagger} a_{\mathbf{q}}+\frac{1}{4} \sum_{\mathbf{p q r s}} \Pi_{\mathbf{p q s r}} a_{\mathbf{p}}^{\dagger} a_{\mathbf{q}}^{\dagger} a_{\mathbf{r}} a_{\mathbf{s}} \\
& =\sum_{\mathbf{p q}}\langle\mathbf{p}|h| \mathbf{q}\rangle a_{\mathbf{p}}^{\dagger} a_{\mathbf{q}}+\frac{1}{4} \sum_{\mathbf{p q r s}}\langle\mathbf{p q}|| \mathbf{s r}\rangle a_{\mathbf{p}}^{\dagger} a_{\mathbf{q}}^{\dagger} a_{\mathbf{r}} a_{\mathbf{s}}
\end{aligned}
$$

projected into the space of all single excitations:

$$
\begin{aligned}
\tilde{A}_{\mathbf{i a j b}} & =\left\langle\Phi_{\mathbf{i}}^{\mathbf{a}}\left|\mathbf{H}_{\mathbf{e l}}\right| \Phi_{\mathbf{j}}^{\mathbf{b}}\right\rangle \\
& =h_{\mathbf{a b}} \delta_{\mathbf{i j}}-h_{\mathbf{j i}} \delta_{\mathbf{a b}}+\delta_{\mathbf{i j}} \sum_{\mathbf{m}} \Pi_{\mathbf{a m b m}}-\delta_{\mathbf{a b}} \sum_{\mathbf{m}} \Pi_{\mathbf{j m i m}}+\Pi_{\mathbf{a j i b}}+E_{H F} \delta_{\mathbf{a b}} \delta_{\mathbf{i j}} .
\end{aligned}
$$

This quantity can be rewritten in terms of the Fock matrix,

$$
\begin{aligned}
F_{\mathbf{p q}} & =h_{\mathbf{p q}}+\sum_{\mathbf{m}} \Pi_{\mathbf{p m q m}} . \\
& =\varepsilon_{\mathbf{p}} \delta_{\mathbf{p q}}
\end{aligned}
$$

The energy of orbital $\mathbf{p}$ is $\varepsilon_{\mathbf{p}}$. By inserting Eqn. 5 into Eqn. 4, we recover the usual CIS theory with "Hamiltonian":

$$
\tilde{A}_{\mathrm{iajb}}=F_{\mathbf{a b}} \delta_{\mathbf{i j}}-F_{\mathbf{j i}} \delta_{\mathbf{a b}}+\Pi_{\mathrm{ajib}}+E_{H F} \delta_{\mathbf{a b}} \delta_{\mathbf{i j}}
$$

The CIS amplitudes $\tilde{\mathbf{X}}$ solve the following eigenvalue problem,

$$
\sum_{\mathbf{b j}} \tilde{A}_{\mathbf{i a j b}} \tilde{X}_{\mathbf{b j}}=\tilde{E} \tilde{X}_{\mathbf{a} \mathbf{i}}
$$


and are normalized such that,

$$
\sum_{\mathbf{a i}} \tilde{X}_{\mathbf{a i}}^{*} \tilde{X}_{\mathbf{a i}}=1
$$

The CIS Hamiltonian is block diagonal in the basis of spin-diabats (singlets and triplets). A CIS singlet state has amplitudes such that

$$
\tilde{X}_{a_{\alpha} i_{\alpha}}=\tilde{X}_{a_{\beta} i_{\beta}} \equiv \frac{1}{\sqrt{2}} s_{a i}
$$

A CIS triplet $\left(m_{s}=0\right)$ state will have amplitudes

$$
\tilde{X}_{a_{\alpha} i_{\alpha}}=-\tilde{X}_{a_{\beta} i_{\beta}} \equiv \frac{1}{\sqrt{2}} t_{a i}^{(0)}
$$

The remaining CIS triplet states will be degenerate with equivalent amplitudes

$$
\begin{aligned}
& \tilde{X}_{a_{\alpha} i_{\beta}} \equiv t_{a i}^{(+1)} \\
& \tilde{X}_{a_{\beta} i_{\alpha}} \equiv t_{a i}^{(-1)}
\end{aligned}
$$

where $t_{a i}^{(0)}=t_{a i}^{(+1)}=t_{a i}^{(-1)}$.

\section{The Breit-Pauli One-Electron Hamiltonian}

A CIS-SOC Hamiltonian extends CIS by including the SOC through the Breit-Pauli Hamiltonian $\mathbf{V}_{\text {SO }}$ (Eqn. 1), which we express here in second quantization notation:

$$
\begin{aligned}
& \mathbf{V}_{\mathbf{S O} x}=-\frac{\alpha_{0}^{2}}{2} \sum_{p q} \tilde{L_{x p q}} \cdot \frac{\hbar}{2}\left(a_{p_{\alpha}}^{\dagger} a_{q_{\beta}}+a_{p_{\beta}}^{\dagger} a_{q_{\alpha}}\right) \\
& \mathbf{V}_{\mathbf{S O} y}=-\frac{\alpha_{0}^{2}}{2} \sum_{p q} \tilde{L}_{y_{p q}} \cdot \frac{\hbar}{2 i}\left(a_{p_{\alpha}}^{\dagger} a_{q_{\beta}}-a_{p_{\beta}}^{\dagger} a_{q_{\alpha}}\right) \\
& \mathbf{V}_{\mathbf{S O} z}=-\frac{\alpha_{0}^{2}}{2} \sum_{p q} \tilde{L}_{z p q} \cdot \frac{\hbar}{2}\left(a_{p_{\alpha}}^{\dagger} a_{q_{\alpha}}-a_{p_{\beta}}^{\dagger} a_{q_{\beta}}\right)
\end{aligned}
$$

Here $\tilde{\mathbf{L}}$ captures the angular moment of an electron moving around all of the different nuclei $A$ with positions $\mathbf{r}_{A}$. For example,

$$
\begin{aligned}
\tilde{L}_{z p q}=\left\langle p\left|\tilde{\mathbf{L}}_{z}\right| q\right\rangle & =\sum_{A} \frac{\hbar Z_{A}}{i}\left\langle p\left|\frac{\left[\left(\mathbf{r}-\mathbf{r}_{A}\right) \times \nabla\right]_{z}}{\left|\mathbf{r}-\mathbf{r}_{A}\right|^{3}}\right| q\right\rangle \\
& =\sum_{A} \frac{\hbar Z_{A}}{i}\left[\left\langle p\left|\frac{\left(x-x_{A}\right)}{\left|\mathbf{r}-\mathbf{r}_{A}\right|^{3}} \frac{\partial}{\partial y}\right| q\right\rangle-\left\langle p\left|\frac{\left(y-y_{A}\right)}{\left|\mathbf{r}-\mathbf{r}_{A}\right|^{3}} \frac{\partial}{\partial x}\right| q\right\rangle\right]
\end{aligned}
$$


One can permute coordinates to recover the $\tilde{\mathbf{L}}_{x}$ and $\tilde{\mathbf{L}}_{y}$ terms. The $\tilde{\mathbf{L}}$ integrals and their derivatives $\tilde{\mathbf{L}}^{[x]}$ are discussed in the appendix.

Finally, for convenience later on, let us define a compact notation for $\mathbf{V}_{\text {So }}$ in the explicit spin basis,

$$
V_{p_{\sigma} q_{\sigma^{\prime}}}=-\frac{\hbar \alpha_{0}^{2}}{4} \tilde{L}_{p_{\sigma} q_{\sigma^{\prime}}}
$$

with the following components for $\tilde{\mathbf{L}}$,

$$
\begin{aligned}
\tilde{L}_{p_{\alpha} q_{\alpha}} & =\tilde{L}_{z_{p q}} \\
\tilde{L}_{p_{\beta} q_{\beta}} & =-\tilde{L}_{z_{p q}} \\
\tilde{L}_{p_{\alpha} q_{\beta}} & =\tilde{L}_{x_{p q}}+\frac{1}{i} \tilde{L}_{y_{p q}} \\
\tilde{L}_{p_{\beta} q_{\alpha}} & =\tilde{L}_{x_{p q}}-\frac{1}{i} \tilde{L}_{y_{p q}}
\end{aligned}
$$

\section{The CIS-SOC Hamiltonian}

The CIS-SOC Hamiltonian is the sum of the $\mathbf{H}_{\mathbf{e l}}$ and $\mathbf{V}_{\text {So }}$ projected into the space of all single excitations:

$$
\begin{aligned}
A_{\mathbf{i a j b}} & =\left\langle\Phi_{\mathbf{i}}^{\mathbf{a}}\left|\mathbf{H}_{\mathrm{el}}+\mathbf{V}_{\mathbf{S O}}\right| \Phi_{\mathbf{j}}^{\mathbf{b}}\right\rangle \\
& =\tilde{A}_{\mathbf{i a j b}}+\left\langle\Phi_{\mathbf{i}}^{\mathbf{a}}\left|\mathbf{V}_{\mathbf{S O}}\right| \Phi_{\mathbf{j}}^{\mathbf{b}}\right\rangle \\
& =\tilde{A}_{\mathbf{i a j b}}+V_{\mathbf{a b}} \delta_{\mathbf{i j}}-V_{\mathbf{j i}} \delta_{\mathbf{a b}}
\end{aligned}
$$

The $\tilde{\mathbf{A}}$ operator in Eqn 18 is the CIS operator (from Eqn. 7).

Let us construct a CIS-SOC stationary state[27], i.e. an eigenstate of $\mathbf{A}$ (not $\tilde{\mathbf{A}}$ ) with energy E:

$$
\sum_{\mathbf{b j}} A_{\mathbf{i a j b}} X_{\mathbf{b j}}=E X_{\mathbf{a i}}
$$

The addition of $\mathbf{V}_{\text {so }}$ mixes singlets and triplets. Thus, such a CIS-SOC eigenstate $|\Psi\rangle$ will have both singlet and triplet contributions,

$$
\begin{aligned}
|\Psi\rangle= & \sum_{\mathbf{a i}} X_{\mathbf{a i}}\left|\Phi_{\mathbf{i}}^{\mathbf{a}}\right\rangle=\sum_{a i} \sum_{\epsilon} X_{a i}^{(\epsilon)}\left|\Phi_{i}^{a(\epsilon)}\right\rangle \\
= & \frac{1}{\sqrt{2}} \sum_{a i} s_{a i}\left(\left|\Phi_{i_{\alpha}}^{a_{\alpha}}\right\rangle+\left|\Phi_{i_{\beta}}^{a_{\beta}}\right\rangle\right)+\frac{1}{\sqrt{2}} \sum_{a i} t_{a i}^{(0)}\left(\left|\Phi_{i_{\alpha}}^{a_{\alpha}}\right\rangle-\left|\Phi_{i_{\beta}}^{a_{\beta}}\right\rangle\right) \\
& +\sum_{a i} t_{a i}^{(+1)}\left|\Phi_{i_{\beta}}^{a_{\alpha}}\right\rangle+\sum_{a i} t_{a i}^{(-1)}\left|\Phi_{i_{\alpha}}^{a_{\beta}}\right\rangle
\end{aligned}
$$


As in standard CIS, the amplitudes are normalized over all contributions,

$$
\sum_{\mathbf{a i}} X_{\mathbf{a i}}^{*} X_{\mathbf{a i}}=\sum_{a i} s_{a i}^{*} s_{a i}+\sum_{a i} \sum_{m_{s}} t_{a i}^{\left(m_{s}\right)^{*}} t_{a i}^{\left(m_{s}\right)}=1
$$

In the explicit spin basis, we can express $\mathbf{X}$ as,

$$
\begin{aligned}
& X_{a_{\alpha} i_{\alpha}}=\frac{1}{\sqrt{2}}\left(s_{a i}+t_{a i}^{(0)}\right) \\
& X_{a_{\beta} i_{\beta}}=\frac{1}{\sqrt{2}}\left(s_{a i}-t_{a i}^{(0)}\right) \\
& X_{a_{\alpha} i_{\beta}}=t_{a i}^{(+1)} \\
& X_{a_{\beta} i_{\alpha}}=t_{a i}^{(-1)}
\end{aligned}
$$

\section{E. Hellmann-Feynman Theory for the CIS-SOC Gradient}

We can now use Hellmann-Feynman Theory to find an analytical gradient for the CISSOC state energy, $E^{[x]}$, given that the CIS-SOC state is an eigenstate of $\mathbf{A}$ :

$$
\begin{aligned}
E^{[x]} & =\sum_{\mathbf{a b i j}}\left(X_{\mathbf{a i}}^{[x] *} A_{\mathbf{i a j b}} X_{\mathbf{b j}}+X_{\mathbf{a i}}^{*} A_{\mathbf{i a j b}} X_{\mathbf{b j}}^{[x]}+X_{\mathbf{a i}}^{*} A_{\mathbf{i a j b}}^{[x]} X_{\mathbf{b j}}\right) \\
& =E \sum_{\mathbf{a i}}\left(X_{\mathbf{a i}}^{[x] *} X_{\mathbf{a} \mathbf{i}}+X_{\mathbf{a i}}^{*} X_{\mathbf{a i}}^{[x]}\right)+\sum_{\mathbf{a b i j}} X_{\mathbf{a} \mathbf{i}}^{*} A_{\mathbf{i a j b}}^{[x]} X_{\mathbf{b j}} \\
& =\sum_{\mathbf{a b i j}} X_{\mathbf{a i}}^{*} A_{\mathbf{i a j b}}^{[x]} X_{\mathbf{b j}}
\end{aligned}
$$

As in Eqn. 18, the standard CIS electronic Hamiltonian can be separated from the new SOC terms,

$$
A_{\mathbf{i a j b}}^{[x]}=\tilde{A}_{\mathbf{i a j b}}^{[x]}+V_{\mathbf{a b}}^{[x]} \delta_{\mathbf{i j}}-V_{\mathbf{j i}}^{[x]} \delta_{\mathbf{a b}}
$$

so that,

$$
E^{[x]}=\sum_{\mathbf{a b i j}} X_{\mathbf{a i}}^{*} \tilde{A}_{\mathbf{i a j b}}^{[x]} X_{\mathbf{b j}}+\sum_{\mathbf{a b i}} X_{\mathbf{a i}}^{*} V_{\mathbf{a b}}^{[x]} X_{\mathbf{b i}}-\sum_{\mathbf{a i j}} X_{\mathbf{a i}}^{*} V_{\mathbf{j i}}^{[x]} X_{\mathbf{a j}}
$$

So far we have been working in a molecular spin orbital basis, but quantum chemistry algorithms are usually designed in the atomic spatial orbital basis to take advantage of realvalued Gaussian-type orbitals with analytic two electron matrix elements. To this end, we will now convert to an atomic spin orbital basis, and then later convert to an atomic spatial orbital basis. 


\section{F. The Atomic Spin Orbital Basis}

Molecular orbitals are linear combinations of AOs with coefficients C. Integrals in the molecular orbital basis, such as $\mathbf{V}_{\mathbf{S O}}$, are sums over integrals calculated in the atomic orbital basis,

$$
V_{\mathrm{pq}}=\sum_{\mu \nu} C_{\mu \mathrm{p}} V_{\mu \nu} C_{\nu \mathbf{q}}
$$

For our purposes below, let us define some important terms in the AO basis,

$$
\begin{aligned}
& S_{\mu \nu} \equiv\langle\boldsymbol{\mu} \mid \boldsymbol{\nu}\rangle \\
& P_{\mu \nu} \equiv \sum_{\mathbf{i}} C_{\mu \mathbf{i}} C_{\nu \mathbf{i}} \\
& \tilde{P}_{\mu \nu} \equiv \sum_{\mathbf{p}} C_{\mu \mathbf{p}} C_{\nu \mathbf{p}}=P_{\mu \nu}+\sum_{\mathbf{a}} C_{\mu \mathbf{a}} C_{\nu \mathbf{a}} \\
& R_{\mu \nu} \equiv \sum_{\mathbf{a i}} C_{\boldsymbol{\mu} \mathbf{a}} X_{\mathbf{a i}} C_{\nu \mathbf{i}} \\
& B_{\mu \nu} \equiv \sum_{\mathbf{a b i}} C_{\boldsymbol{\mu} \mathbf{a}} X_{\mathbf{a} \mathbf{i}}^{*} X_{\mathbf{b i}} C_{\nu \mathbf{b}}-\sum_{\mathbf{a i j}} C_{\boldsymbol{\mu} \mathbf{j}} X_{\mathbf{a j}} X_{\mathbf{a} \mathbf{i}}^{*} C_{\nu \mathbf{i}}
\end{aligned}
$$

The first three equations 27-29) define the overlap matrix, the ground state density matrix and the formal inverse $\mathbf{S}^{-1}$, respectively. Eqn. 30 is the CIS amplitudes in the AO basis, also known as the transition density. Eqn. 31 is very similar to the difference density matrix, but the second term is transposed.

In order to convert the derivatives in Eqn. 25 from the MO basis to the AO basis, we will use the $\mathbf{V}_{\mathbf{S O}}{ }^{[x]}$ term as an example. To start, we apply the derivative operator to Eqn. 26.

$$
V_{\mathbf{p q}}^{[x]}=\sum_{\mu \nu} V_{\mu \nu}^{[x]} C_{\mu \mathbf{p}} C_{\nu \mathbf{q}}+\sum_{\mu \nu} V_{\mu \nu}\left(C_{\mu \mathbf{p}}^{[x]} C_{\nu \mathbf{q}}+C_{\mu \mathbf{p}} C_{\nu \mathbf{q}}^{[x]}\right)
$$

The $V_{\boldsymbol{\mu}}^{[x]}$ term is easily dependent on the $\tilde{\mathbf{L}}^{[x]}$ integrals, which are directly available, but the $C^{[x]}$ terms are not. Others have derived the form of the $C^{[x]}$ derivatives [28] and we summarize the main points here. The molecular orbital coefficients depend on the overlap of the atomic orbitals $(\mathbf{S})$ and the rotation matrix between the virtual and occupied space $\left(\Theta_{b i}\right)$, so the derivative can we written,

$$
C_{\boldsymbol{\mu} \mathbf{p}}^{[x]}=\sum_{\gamma \boldsymbol{\lambda}} \frac{\partial C_{\boldsymbol{\mu p}}}{\partial S_{\gamma \boldsymbol{\lambda}}} S_{\gamma \boldsymbol{\lambda}}^{[x]}+\sum_{\mathbf{c k}} \frac{\partial C_{\boldsymbol{\mu p}}}{\partial \Theta_{\mathbf{c k}}} \Theta_{\mathbf{c k}}^{[x]}
$$


The form of the partial derivatives can be written

$$
\begin{aligned}
& \frac{\partial C_{\boldsymbol{\mu p}}}{\partial S_{\gamma \lambda}}=-\frac{1}{2} \tilde{P}_{\mu \gamma} C_{\lambda \mathbf{p}} \\
& \frac{\partial C_{\boldsymbol{\mu p}}}{\partial \Theta_{\mathrm{ck}}}=C_{\boldsymbol{\mu k}} \delta_{\mathbf{c p}}-C_{\boldsymbol{\mu c}} \delta_{\mathbf{k p}}
\end{aligned}
$$

Inserting Eqns. 34 and 35 into Eqn. 33, we find

$$
C_{\mu \mathbf{p}}^{[x]}=-\frac{1}{2} \sum_{\gamma \lambda} \tilde{P}_{\mu \gamma} S_{\gamma \lambda}^{[x]} C_{\lambda \mathbf{p}}+\sum_{\mathbf{c k}}\left(C_{\boldsymbol{\mu k}} \delta_{\mathbf{c p}}-C_{\boldsymbol{\mu c}} \delta_{\mathbf{k p}}\right) \Theta_{\mathbf{c k}}^{[x]}
$$

Finally, inserting Eqn. 36 into Eqn. 32 gives

$$
\begin{aligned}
V_{\mathbf{p q}}^{[x]}= & \sum_{\mu \nu} V_{\mu \nu}^{[x]} C_{\boldsymbol{\mu p}} C_{\boldsymbol{\nu} \mathbf{q}} \\
& -\frac{1}{2} \sum_{\mu \nu \gamma \lambda} S_{\gamma \lambda}^{[x]} V_{\mu \nu}\left(C_{\boldsymbol{\lambda} \mathbf{p}} C_{\nu \mathbf{q}} \tilde{P}_{\gamma \mu}+C_{\boldsymbol{\lambda} \mathbf{q}} C_{\boldsymbol{\mu} \mathbf{p}} \tilde{P}_{\nu \gamma}\right) \\
& +\sum_{\mu \nu \mathbf{c k}} \Theta_{\mathbf{c k}}^{[x]} V_{\mu \nu}\left(\left(C_{\mu \mathbf{k}} \delta_{\mathbf{c p}}-C_{\mu \mathbf{c}} \delta_{\mathbf{k p}}\right) C_{\nu \mathbf{q}}+C_{\mu \mathbf{p}}\left(C_{\nu \mathbf{k}} \delta_{\mathbf{c q}}-C_{\nu \mathbf{c}} \delta_{\mathbf{k q}}\right)\right)
\end{aligned}
$$

Eqn. 37 is our final form for $\mathbf{V}_{\mathbf{S O}}{ }^{[x]}$ in the atomic spin orbital basis. The same steps can be applied to the standard CIS terms, the Fock term derivatives and two electron term derivatives. See reference [29].

\section{G. The CIS-SOC gradient in the Atomic Spin Orbital Basis}

Now we have all the tools required to write the CIS-SOC gradient in the atomic spin orbital basis. In an atomic spin orbital basis, $E^{[x]}$ can naturally be written as the sum of four terms:

$$
E^{[x]}=E_{A}^{[x]}+E_{V}^{[x]}+\sum_{\mathbf{c k}} \Theta_{\mathbf{c k}}^{[x]}\left(Y_{\mathbf{c k}}-Y_{\mathbf{c k}}^{S O C}\right)+E_{H F}^{[x]}
$$

Let us now define these terms.

The first term $E_{A}^{[x]}$ is the standard CIS contribution without the ground state derivative 
$E_{H F}^{[x]}$ and without including orbital relaxation [29, 30]:

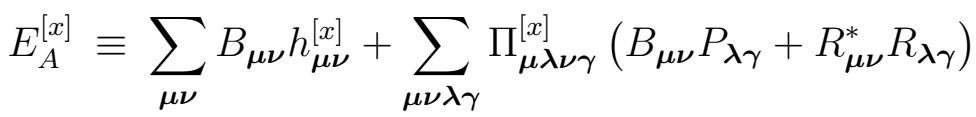

$$
\begin{aligned}
& -\frac{1}{2} \sum_{\mu \nu \gamma \boldsymbol{\lambda}} S_{\gamma \lambda}^{[x]} \tilde{P}_{\gamma \boldsymbol{\mu}}\left(B_{\boldsymbol{\lambda} \boldsymbol{\nu}}+B_{\boldsymbol{\nu} \boldsymbol{\lambda}}\right) F_{\boldsymbol{\nu} \boldsymbol{\mu}} \\
& -\frac{1}{2} \sum_{\boldsymbol{\mu} \boldsymbol{\nu} \boldsymbol{\lambda} \boldsymbol{\delta} \boldsymbol{\omega}} S_{\boldsymbol{\delta} \boldsymbol{\omega}}^{[x]} \tilde{P}_{\boldsymbol{\lambda} \boldsymbol{\delta}} P_{\boldsymbol{\omega} \gamma}\left(B_{\boldsymbol{\mu} \boldsymbol{\nu}}+B_{\boldsymbol{\nu} \boldsymbol{\mu}}\right) \Pi_{\boldsymbol{\mu} \boldsymbol{\lambda} \boldsymbol{\nu} \gamma} \\
& -\frac{1}{2} \sum_{\boldsymbol{\mu} \boldsymbol{\nu} \boldsymbol{\lambda} \boldsymbol{\delta} \boldsymbol{\omega}} S_{\boldsymbol{\delta} \boldsymbol{\omega}}^{[x]} \tilde{P}_{\boldsymbol{\mu} \boldsymbol{\delta}}\left(R_{\boldsymbol{\omega} \boldsymbol{\nu}}^{*} R_{\boldsymbol{\gamma} \boldsymbol{\lambda}}+R_{\boldsymbol{\nu} \boldsymbol{\omega}}^{*} R_{\boldsymbol{\lambda} \gamma}\right) \Pi_{\boldsymbol{\mu} \boldsymbol{\lambda} \boldsymbol{\gamma}} \\
& -\frac{1}{2} \sum_{\boldsymbol{\mu} \boldsymbol{\nu} \boldsymbol{\lambda} \boldsymbol{\delta} \boldsymbol{\omega}} S_{\boldsymbol{\delta} \boldsymbol{\omega}}^{[x]} \tilde{P}_{\gamma \boldsymbol{\delta}}\left(R_{\boldsymbol{\mu} \boldsymbol{\nu}}^{*} R_{\boldsymbol{\omega} \boldsymbol{\lambda}}+R_{\boldsymbol{\nu} \boldsymbol{\mu}}^{*} R_{\boldsymbol{\lambda} \boldsymbol{\omega}}\right) \Pi_{\boldsymbol{\mu} \boldsymbol{\lambda} \boldsymbol{\gamma}}
\end{aligned}
$$

The second term is the contribution from $\mathbf{V}_{\text {So }}$ without including orbital relaxation[31]:

$$
\begin{aligned}
E_{V}^{[x]} \equiv & -\frac{\hbar \alpha_{0}^{2}}{4} \sum_{\boldsymbol{\mu} \boldsymbol{\nu}} \tilde{L}_{\boldsymbol{\mu \nu}}^{[x]} B_{\boldsymbol{\mu \nu}} \\
& +\frac{\hbar \alpha_{0}^{2}}{8} \sum_{\boldsymbol{\mu \nu} \boldsymbol{\gamma} \boldsymbol{\lambda}} S_{\gamma \lambda}^{[x]} \tilde{P}_{\gamma \boldsymbol{\mu}}\left(\tilde{L}_{\boldsymbol{\mu \nu}} B_{\boldsymbol{\lambda} \boldsymbol{\nu}}+\tilde{L}_{\boldsymbol{\nu} \boldsymbol{\mu}} B_{\boldsymbol{\nu} \boldsymbol{\lambda}}\right)
\end{aligned}
$$

The third term is the gradient component arising from orbital relaxation. Here, again, there are two terms. The $\mathbf{Y}$ term arises from standard CIS theory [29].

$$
\begin{aligned}
Y_{\mathbf{c k}} \equiv & \sum_{\mu \nu \lambda \gamma} C_{\lambda \mathbf{c}} C_{\gamma \mathrm{k}}\left(B_{\mu \nu}+B_{\nu \mu}\right) \Pi_{\mu \lambda \nu \gamma} \\
& +\sum_{\mathbf{j} \mu \nu \lambda \gamma} C_{\nu \mathrm{j}} C_{\mu \mathrm{k}}\left(X_{\mathbf{c j}}^{*} R_{\gamma \boldsymbol{\lambda}}+X_{\mathbf{c j}} R_{\gamma \lambda}^{*}\right) \Pi_{\mu \lambda \nu \gamma} \\
& -\sum_{\mathbf{b} \mu \nu \lambda \gamma} C_{\nu \mathbf{c}} C_{\mu \mathrm{b}}\left(R_{\gamma \lambda} X_{\mathbf{b k}}^{*}+R_{\gamma \lambda}^{*} X_{\mathbf{b k}}\right) \Pi_{\mu \lambda \nu \gamma}
\end{aligned}
$$

The $\mathbf{Y}^{S O C}$ term arises from the SOC.

$$
\begin{aligned}
Y_{\mathbf{c k}}^{S O C} \equiv & -\sum_{\mathbf{a i}}\left(X_{\mathbf{c i}}^{*} X_{\mathbf{a i}} V_{\mathbf{k a}}+X_{\mathbf{c i}} X_{\mathbf{a i}}^{*} V_{\mathbf{a k}}\right) \\
& -\sum_{\mathbf{a i}}\left(V_{\mathbf{c i}} X_{\mathbf{a i}}^{*} X_{\mathbf{a k}}+V_{\mathbf{i c}} X_{\mathbf{a i}} X_{\mathbf{a k}}^{*}\right)
\end{aligned}
$$

The fourth term is the ground state derivative, $E_{H F}^{[x]}$.

\section{H. The Atomic Spatial Orbital Basis}

At this point, we have used only the usual tricks to evaluate the CIS gradient, from Ref. [29]. The last and final step is to integrate over the spin degrees of freedom and evaluate 
the gradient in Eqns. 38,42 in terms of atomic spatial orbitals. This requires explicit spin information in our integrals.

We emphasize that most of the atomic orbital terms do not mix different spins. The explicit spin information for these terms can be expressed easily as:

$$
\begin{aligned}
C_{\mu_{\sigma} p_{\sigma^{\prime}}} & =C_{\mu p} \delta_{\sigma \sigma^{\prime}} \\
S_{\mu_{\sigma} \nu_{\sigma^{\prime}}} & =S_{\mu \nu} \delta_{\sigma \sigma^{\prime}} \\
P_{\mu_{\sigma} \nu_{\sigma^{\prime}}} & =\delta_{\sigma \sigma^{\prime}} \sum_{i} C_{\mu i} C_{\nu i} \\
\tilde{P}_{\mu_{\sigma} \nu_{\sigma^{\prime}}} & =\delta_{\sigma \sigma^{\prime}} \sum_{p} C_{\mu p} C_{\nu p}
\end{aligned}
$$

When we integrate over spin, these terms will not contain any spin information. The terms in an atomic spatial orbital basis that do have unique spin information are,

$$
\begin{aligned}
R_{\mu_{\sigma} \nu_{\sigma^{\prime}}} & =\sum_{a i} C_{\mu a} X_{a_{\sigma} i_{\sigma^{\prime}}} C_{\nu i} \\
B_{\mu_{\sigma} \nu_{\sigma^{\prime}}} & =\sum_{a b i} \sum_{\sigma^{\prime \prime}} C_{\mu a} X_{a_{\sigma} i_{\sigma^{\prime \prime}}}^{*} X_{b_{\sigma^{\prime}} i_{\sigma^{\prime \prime}}} C_{\nu b}-\sum_{a i j} \sum_{\sigma^{\prime \prime}} C_{\mu j} X_{a_{\sigma^{\prime \prime}} j_{\sigma}} X_{a_{\sigma^{\prime \prime}} i_{\sigma^{\prime}}}^{*} C_{\nu i}
\end{aligned}
$$

Now, the $\boldsymbol{\Pi}$ tensor might appear more complicated than necessary in the explicit spin basis. After all, $\Pi$ is block diagonal in the spin-diabat basis, and so it will be convenient to define the two different forms (depending on spin). When we integrate over singlet diabatic states, the tensor takes the form,

$$
\Pi_{\mu \lambda \nu \gamma}^{(s)} \equiv 2\langle\mu \lambda \mid \nu \gamma\rangle-\langle\mu \lambda \mid \gamma \nu\rangle
$$

When we integrate over triplet diabatic states, the tensor takes the form,

$$
\Pi_{\mu \lambda \nu \gamma}^{(t)} \equiv-\langle\mu \lambda \mid \gamma \nu\rangle
$$

In this framework, the Fock matrix has a contribution from the singlet form of $\Pi$, i.e.

$$
F_{\mu_{\sigma} \nu_{\sigma^{\prime}}}=\delta_{\sigma \sigma^{\prime}}\left(h_{\mu \nu}+\sum_{\lambda \gamma} P_{\lambda \gamma} \Pi_{\mu \lambda \nu \gamma}^{(s)}\right)
$$

For use below, we will also define $\mathbf{R}$ and $\mathbf{B}$ in the spin-diabatic basis,

$$
\begin{aligned}
R_{\mu \nu}^{(\epsilon)} & \equiv \sum_{a i} C_{\mu a} X_{a i}^{(\epsilon)} C_{\nu i} \\
B_{\mu \nu}^{(\epsilon)} & \equiv \sum_{a b i} C_{\mu a} X_{a i}^{(\epsilon) *} X_{b i}^{(\epsilon)} C_{\nu b}-\sum_{a i j} C_{\mu j} X_{a j}^{(\epsilon) *} X_{a i}^{(\epsilon)} C_{\nu i}
\end{aligned}
$$


Here, $\epsilon \in\left\{s, m_{s}=-1,0,+1\right\}$.

The last matrix element required in a spatial orbital basis is the rotation matrix between the virtual and occupied space. Given that we assume the Hartree-Fock ground state will always be a closed shell singlet, the molecular orbital basis never mixes spin, so that

$$
\Theta_{c_{\sigma} k_{\sigma^{\prime}}}=\Theta_{c k} \delta_{\sigma \sigma^{\prime}}
$$

\section{The CIS-SOC gradient in the Atomic Spatial Orbital Basis}

Using the above equations, we can construct a final working analytical gradient:

$$
E^{[x]}=\sum_{\epsilon} E_{A}^{(\epsilon)}{ }^{[x]}+E_{V}^{[x]}+\sum_{c k} \Theta_{c k}^{[x]}\left(\sum_{\epsilon} Y_{c k}^{(\epsilon)}-\sum_{\sigma} Y_{c_{\sigma} k_{\sigma}}^{S O C}\right)+E_{H F}^{[x]}
$$

As stated above, the electronic Hamiltonian components are all block diagonal in the spindiabatic basis, and we see the same behavior with the gradient. This means we can sum over the independent contributions of the various spin-diabats $\left(\epsilon \in\left\{s, m_{s}=-1,0,+1\right\}\right)$ :

$$
\begin{aligned}
E_{A}^{(\epsilon)} \equiv & \sum_{\mu \nu} B_{\mu \nu}^{(\epsilon)} h_{\mu \nu}^{[x]}+\sum_{\mu \nu \lambda \gamma}\left(\Pi_{\mu \lambda \nu \gamma}^{(s)[x]} B_{\mu \nu}^{(\epsilon)} P_{\lambda \gamma}+\Pi_{\mu \lambda \nu \gamma}^{(\epsilon)[x]} R_{\mu \nu}^{(\epsilon) *} R_{\lambda \gamma}^{(\epsilon)}\right) \\
& -\frac{1}{2} \sum_{\mu \nu \gamma \lambda} S_{\gamma \lambda}^{[x]} \tilde{P}_{\gamma \mu}\left(B_{\lambda \nu}^{(\epsilon)}+B_{\nu \lambda}^{(\epsilon)}\right) F_{\nu \mu} \\
& -\frac{1}{2} \sum_{\mu \nu \lambda \gamma \delta \omega} S_{\delta \omega}^{[x]} \tilde{P}_{\lambda \delta} P_{\omega \gamma}\left(B_{\mu \nu}^{(\epsilon)}+B_{\nu \mu}^{(\epsilon)}\right) \Pi_{\mu \lambda \nu \gamma}^{(s)} \\
& -\frac{1}{2} \sum_{\mu \nu \lambda \gamma \delta \omega} S_{\delta \omega}^{[x]} \tilde{P}_{\mu \delta}\left(R_{\omega \nu}^{(\epsilon) *} R_{\gamma \lambda}^{(\epsilon)}+R_{\nu \omega}^{(\epsilon) *} R_{\lambda \gamma}^{(\epsilon)}\right) \Pi_{\mu \lambda \nu \gamma}^{(\epsilon)} \\
& -\frac{1}{2} \sum_{\mu \nu \lambda \gamma \delta \omega} S_{\delta \omega}^{[x]} \tilde{P}_{\gamma \delta}\left(R_{\mu \nu}^{(\epsilon) *} R_{\omega \lambda}^{(\epsilon)}+R_{\nu \mu}^{(\epsilon) *} R_{\lambda \omega}^{(\epsilon)}\right) \Pi_{\mu \lambda \nu \gamma}^{(\epsilon)}
\end{aligned}
$$

Similarly, we can define the $\mathbf{Y}^{(\epsilon)}$ term,

$$
\begin{aligned}
Y_{c k}^{(\epsilon)} \equiv & \sum_{\mu \nu \lambda \gamma} C_{\lambda c} C_{\gamma k}\left(B_{\mu \nu}^{(\epsilon)}+B_{\nu \mu}^{(\epsilon)}\right) \Pi_{\mu \lambda \nu \gamma}^{(s)} \\
& +\sum_{j \mu \nu \lambda \gamma} C_{\nu j} C_{\mu k}\left(X_{c j}^{(\epsilon) *} R_{\gamma \lambda}^{(\epsilon)}+X_{c j}^{(\epsilon)} R_{\gamma \lambda}^{(\epsilon) *}\right) \Pi_{\mu \lambda \nu \gamma}^{(\epsilon)} \\
& -\sum_{b \mu \nu \lambda \gamma} C_{\nu c} C_{\mu b}\left(R_{\gamma \lambda}^{(\epsilon)} X_{b k}^{(\epsilon) *}+R_{\gamma \lambda}^{(\epsilon) *} X_{b k}^{(\epsilon)}\right) \Pi_{\mu \lambda \nu \gamma}^{(\epsilon)}
\end{aligned}
$$


For the $E_{V}^{[x]}$ term, we cannot simplify to spin-diabats, so we integrate over the spin degrees of freedom and express the results explicitly here,

$$
\begin{aligned}
E_{V}^{[x]}= & -\frac{\hbar \alpha_{0}^{2}}{4} \sum_{\mu \nu} \sum_{\sigma \sigma^{\prime}} \tilde{L}_{\mu_{\sigma} \nu_{\sigma^{\prime}}}^{[x]} B_{\mu_{\sigma} \nu_{\sigma^{\prime}}} \\
& +\frac{\hbar \alpha_{0}^{2}}{8} \sum_{\mu \nu \gamma \lambda} \sum_{\sigma \sigma^{\prime}} S_{\gamma \lambda}^{[x]} \tilde{P}_{\gamma \mu}\left(\tilde{L}_{\mu_{\sigma} \nu_{\sigma^{\prime}}} B_{\lambda_{\sigma} \nu_{\sigma^{\prime}}}+\tilde{L}_{\nu_{\sigma^{\prime}} \mu_{\sigma}} B_{\nu_{\sigma^{\prime}} \lambda_{\sigma}}\right)
\end{aligned}
$$

The $\tilde{\mathbf{L}}$ integrals are defined in this basis in Eqn. 17. The $\mathbf{B}$ terms are defined in Eqn. 48 , using the $\mathbf{X}$ from Eqn. 22, From Eqn. 54, we know that the $\boldsymbol{\Theta}^{[x]}$ term restricts the spin of $\mathbf{Y}^{S O C}$, so we can write this quantity explicitly as,

$$
\begin{aligned}
Y_{c_{\sigma} k_{\sigma}}^{S O C} \equiv & -\sum_{a i} \sum_{\sigma^{\prime} \sigma^{\prime \prime}}\left(X_{c_{\sigma} i_{\sigma^{\prime}}}^{*} X_{a_{\sigma^{\prime \prime}} i_{\sigma^{\prime}}} V_{k_{\sigma} a_{\sigma^{\prime \prime}}}+X_{c_{\sigma} i_{\sigma^{\prime}}} X_{a_{\sigma^{\prime \prime}} i_{\sigma^{\prime}}}^{*} V_{a_{\sigma^{\prime \prime}} k_{\sigma}}\right) \\
& -\sum_{a i} \sum_{\sigma^{\prime} \sigma^{\prime \prime}}\left(V_{c_{\sigma} i_{\sigma^{\prime}}} X_{a_{\sigma^{\prime \prime}} i_{\sigma^{\prime}}}^{*} X_{a_{\sigma^{\prime \prime}} k_{\sigma}}+V_{i_{\sigma^{\prime}} c_{\sigma}} X_{a_{\sigma^{\prime \prime}} i_{\sigma^{\prime}}} X_{a_{\sigma^{\prime \prime}} k_{\sigma}}^{*}\right)
\end{aligned}
$$

All of the terms in Eqns. 55,59 are available in standard quantum chemistry software, except the $\tilde{\mathbf{L}}$ integrals and derivatives, which we have implemented. As is standard in gradient theory, $\boldsymbol{\Theta}^{[\mathbf{x}]}$ is not calculated directly, but rather through the coupled-perturbed Hartree-Fock theory (CPHF) with a Z-vector scheme[32 34].

\section{RESULTS}

In a development version of the Q-Chem software package 35], we have implemented our CIS-SOC algorithm and the nuclear gradients of these CIS-SOC states (Eqns. 5559. Our reference molecule is ethene at the $\mathrm{S}_{2} / \mathrm{T}_{4}$ crossing geometry as calculated at the $\mathrm{HF} / 6-31 \mathrm{G}^{* *}$ level of theory.

\section{A. States at an Intersystem Crossing}

We have made use of a Davidson-inspired iterative diagonalization scheme for finding stationary states of the complex CIS-SOC Hamiltonian (Eqn. 18) and satisfy Eqn. 19 [36, 37]. The method searches for the lowest eigenvalues of a matrix in a subspace of the full basis. The number of iterations required to converge the eigenvalues depends greatly on the initial guess of the subspace. Calculating standard CIS states scales formally as $O\left(N^{2}\right)$ where 
$N$ is the size of the matrix. When we mix singlets and triplets, one would naively expect that the computational cost of CIS-SOC would go up by a factor of 16 relative to standard CIS; and when one considers the transition from a real to complex Hermitian Hamiltonian, the cost should go up by another factor of 2 (for a total factor of 32 times the cost). Perhaps not surprisingly, we have found that the cost of CIS-SOC is reduced dramatically if we use standard CIS singlets and triplets as an initial guess subspace.

For our small example of ethene, all calculations were run in serial. When one uses 5 singlet and 5 triplet standard CIS states as the initial guess (20 spin-diabats in total), the CIS-SOC calculation requires only 4 iterations. Table 1 shows that the total calculation time for this example is less than the cost of standard CIS.

\begin{tabular}{|c|c|c|c|}
\hline & Wall Time & PU Tir & cations \\
\hline CIS & $5.6 \mathrm{~s}$ & $3.3 \mathrm{~s}$ & 29 \\
\hline CIS-SOC & $3.8 \mathrm{~s}$ & $3.5 \mathrm{~s}$ & 4 \\
\hline Total & $9.4 \mathrm{~s}$ & $6.8 \mathrm{~s}$ & - \\
\hline
\end{tabular}

TABLE I: Calculation timing at the $\mathrm{S}_{2} / \mathrm{T}_{4}$ crossing geometry of ethene. For this case, the CIS-SOC calculation does not add much overall cost to a CIS calculation.

Figure 2 demonstrates the effect of including the SOC at the $\mathrm{S}_{2} / \mathrm{T}_{4}$ crossing geometry. When one rotates ethene along its torsion angle, the CIS energies of the $\mathrm{S}_{2}$ and $\mathrm{T}_{4}$ states cross at $\theta_{c} \equiv 14.635^{\circ}$ and $\tilde{E}\left(\theta_{c}\right)=77.675 \mathrm{E}_{h}$. With SOC, the $\mathrm{S}_{2}$ singlet diabat and the three $\mathrm{T}_{4}$ triplet diabats mix, generating two mixed spin-adiabats and two degenerate triplet adiabats.

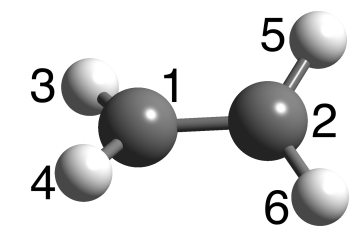

FIG. 1: A schematic representation of ethene twisting along its torsion angle. The numbers correspond to the labels used in the appendix. 


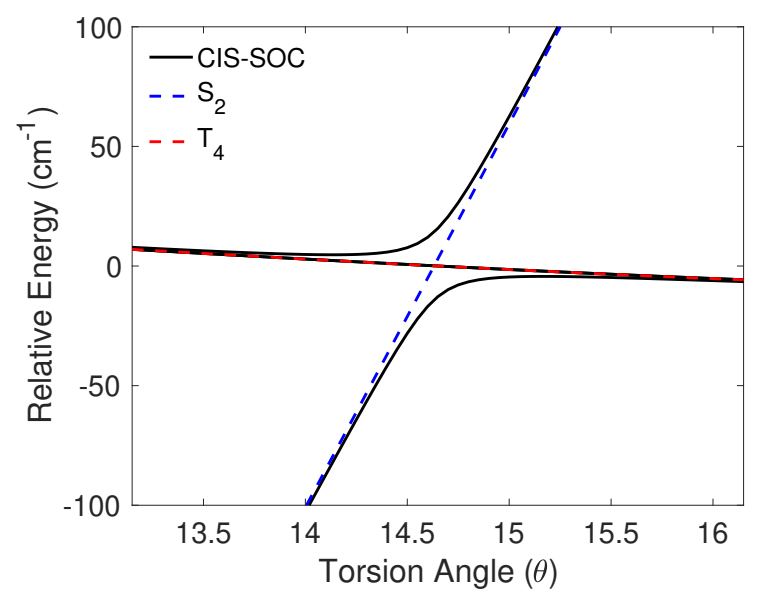

FIG. 2: The CIS and CIS-SOC potential energy surfaces at the $\mathrm{S}_{2} / \mathrm{T}_{4}$ intersystem crossing of ethene as a function of the torsion angle relative to the energy of the crossing point of the CIS states $(\tilde{E}(\theta=14.635)=77.676 \mathrm{Ha})$. The blue (red) dashed line represents the singlet (triplet) CIS spin-diabat. The black lines are the CIS-SOC spin-adiabats (states $11-14$ ). The crossing point geometry is given in the appendix.

\section{B. Comparison to Finite Difference}

For the ethene case in Fig. 2, we have used a five point stencil to calculate energy gradients by finite difference at the crossing point for $\mathrm{H} 3$ and $\mathrm{C} 1$.

\begin{tabular}{|c|c|c|c|c|}
\hline \multirow[b]{2}{*}{ Atom } & \multicolumn{2}{|c|}{$\begin{array}{l}\text { Finite Difference } \\
\qquad\left(\mathrm{E}_{h} \cdot a_{0}^{-1}\right)\end{array}$} & \multicolumn{2}{|r|}{$\begin{array}{l}\text { Analytical } \\
\left(\mathrm{E}_{h} \cdot a_{0}^{-1}\right)\end{array}$} \\
\hline & $\mathrm{y}$ & $\mathrm{Z}$ & $\mathrm{x}$ & y $\quad$ z \\
\hline H3 & -0.005300 .01884 & 0.00761 & -0.00530 & 0.018840 .00761 \\
\hline $\mathrm{C} 1$ & -0.127670 .00000 & 0.00000 & -0.12767 & 70.000000 .00000 \\
\hline
\end{tabular}

TABLE II: Gradient of CIS-SOC state 14 in atomic units. Note that the analytical results agree with finite difference.

\section{DISCUSSION AND CONCLUSION}

We have derived and implemented analytic gradients for the spin-adiabatic states corresponding to a CIS Hamiltonian when we include SOC. As argued in the introduction, there 
are many applications for which we believe this theory will be relevant, especially surface hopping nonadiabatic dynamics. Nevertheless, the approach taken here has been by brute force, and one might wonder if the math to get to Eqns. 55,59 was really necessary? After all, if we want spin-adiabats, one must wonder why we have not implemented the most obvious alternative algorithm? Naively, we could calculate the singlet and triplet states directly and then couple a smaller subset together through SOC [11, 26, 38, 40]. With such an approach, however, we emphasize that one cannot apply Hellmann-Feynman theorem, so that for a derivative, one must calculate explicitly how the singlet and triplet states change as a function of nuclear coordinates[26]. Furthermore, using a Z-vector to address such changes may be unstable due to high energy intruder states or [41]. By contrast, our presented method with spin-adiabats avoids all such difficulties; while we spend somewhat more time on matrix diagonalization, we spend far less time on the gradient.

Looking forward, one big question is how to transfer all this technology from CIS to TDDFT. After all, TD-DFT is known to correct the orbital energies relative to Hartree-Fock and CIS, and yield much better excitation energies. Of course, there are problems with charge transfer states, but using TD-DFT, many problems can be resolved if one uses a range corrected functional [42 54]. Now, when calculating spin-adiabats with TD-DFT, the most obvious difficulty is how to treat the exchange-correlated functional which looks like a two-electron matrix element in the singles-singles block. However, for such an operator to be nonzero all electrons must have the same spin. Thus, formally, one should recover different excitation energies for the $m_{s}=1$ or -1 triplets relative to the $m_{s}=0$ triplet (and the latter is more accurate). Nevertheless,one solution to this quandary would be to simply include the same exchange-correlation functional for all triplet terms, which will necessarily maintain the normal spin degeneracy and should produce better excitation energies.

Finally, in the future, one can now imagine several applications worth exploring. With a fast enough ab initio code, an obvious target is the photophysics of benzaldehyde and benzophenone and the resulting ISC and phosphorescence [55, 56]. More generally, there have recently been interesting experiments done by Vinogradov and coworkers, where two singlets converted to triplets in platinum complexes and there have been few calculations [57]. These are just two out of many possible future applications. 


\section{ACKNOWLEDGEMENT}

We thank Yihan Shao for helpful conversations. This material is based upon work supported by the the National Science Foundation under Grant No. CHE-1764365 and the National Science Foundation Graduate Research Fellowship Program under Grant No. DGE1321851.

\section{APPENDIX}

\section{A. Geometry of the $\mathbf{S}_{2} / \mathbf{T}_{4}$ Crossing Point of Ethene}

The $\mathrm{S}_{2} / \mathrm{T}_{4}$ crossing point was found by optimizing the geometry of the ground state and rotating two geminal hydrogens. Table III reports the geometry at the crossing point.

\begin{tabular}{c|ccc} 
Atom & $\mathrm{x}(\AA)$ & $\mathrm{y}(\AA)$ & $\mathrm{z}(\AA)$ \\
\hline $\mathrm{C}(1)$ & 0.658180 & 0.000000 & 0.000000 \\
$\mathrm{C}(2)$ & -0.658180 & 0.000000 & -0.000000 \\
$\mathrm{H}(3)$ & 1.224535 & -0.907854 & -0.116580 \\
$\mathrm{H}(4)$ & 1.224537 & 0.907854 & 0.116580 \\
$\mathrm{H}(5)$ & -1.224537 & -0.907854 & 0.116580 \\
$\mathrm{H}(6)$ & -1.224537 & 0.907854 & -0.116580
\end{tabular}

TABLE III: Geometry of ethene at the $\mathrm{S}_{2} / \mathrm{T}_{4}$ Crossing Point

\section{B. The Spin-Orbit Coupling Integrals and Their Derivatives}

The CIS-SOC gradient requires access to the derivatives of the spin-orbit integrals in the atomic orbital basis, $\tilde{\mathbf{L}}^{[x]}$ in Eqn. 58. To this end, we have implemented King and Furlani's algorithm [58] for spin-orbit integrals and extended the algorithm to integral nuclear derivatives. King and Furlani present recursion relations that express the spin-orbit multidimensional integrals as products of one dimensional integrals summed over the roots of the Rys polynomial [58]. Here, we will quickly outline the relevant formulas to evaluate the nuclear derivatives of the integrals. 


\section{Spin-orbit coupling in terms of nuclear attraction integrals}

Atomic orbitals are linear combinations of Guassian primitive functions of the form,

$$
\left|\eta_{a}\right\rangle=\left(x-x_{a}\right)^{n_{a}^{x}}\left(y-y_{a}\right)^{n_{a}^{y}}\left(z-z_{a}\right)^{n_{a}^{z}} e^{-\alpha_{a} \mathbf{r}_{a}^{2}}
$$

Here $\mathbf{r}_{a}=\left|r-r_{a}\right|$ is the distance from the center of the Gaussian and the exponents $\left\{n^{x}, n^{y}, n^{z}\right\}$ are the angular momentum for each coordinate and are elements of the natural numbers $\mathbb{N}_{0}$. Consider the contribution of an atom $C$ to the $\tilde{L}_{z}$ term of the SOC (of course, one can permute coordinates to recover the $\tilde{L}_{x}$ and $\tilde{L}_{y}$ terms).

$$
\left\langle\eta_{a}\left|\tilde{L}_{z, C}\right| \eta_{b}\right\rangle=\frac{\hbar Z_{C}}{i}\left[\int \eta_{a} \frac{\left(x-x_{C}\right)}{\mathbf{r}_{\mathbf{C}^{3}}} \frac{\partial \eta_{b}}{\partial y} d r-\int \eta_{a} \frac{\left(y-y_{C}\right)}{\mathbf{r}_{\mathbf{C}}{ }^{3}} \frac{\partial \eta_{b}}{\partial x} d r\right]
$$

King and Furlani convert the integrals with $1 / \mathbf{r}^{3}$ dependence to nuclear attraction integrals $(1 / \mathbf{r}$ dependence) of the form,

$$
\left\langle\eta_{a}\left|L_{z, C}\right| \eta_{b}\right\rangle=\frac{\hbar Z_{C}}{i}\left[\left\langle\eta_{a}^{[x]}\left|\frac{1}{\mathbf{r}_{\mathbf{C}}}\right| \eta_{b}^{[y]}\right\rangle-\left\langle\eta_{a}^{[y]}\left|\frac{1}{\mathbf{r}_{\mathbf{C}}}\right| \eta_{b}^{[x]}\right\rangle\right]
$$

where the derivative of the Gaussian can be defined as

$$
\left|\eta_{a}^{[x]}\right\rangle \equiv \frac{\partial \eta_{a}}{\partial x}=n_{a}^{x}\left|\eta_{a}^{x-}\right\rangle-2 \alpha_{a}\left|\eta_{a}^{x+}\right\rangle
$$

using short hand for increasing or decreasing the angular momentum in a coordinate $x$ :

$$
\left|\eta_{a}^{x \pm}\right\rangle \equiv\left(x-x_{a}\right)^{n_{a}^{x} \pm 1}\left(y-y_{a}\right)^{n_{a}^{y}}\left(z-z_{a}\right)^{n_{a}^{z}} e^{-\alpha_{a} \mathbf{r}_{a}^{2}}
$$

\section{Extension to the Nuclear Derivative}

The nuclear derivative for the $q$ coordinate of atom $D$ of $\tilde{\mathbf{L}}_{z, C}$ is

$$
\left\langle\eta_{a}\left|L_{z, C}\right| \eta_{b}\right\rangle^{\left[q_{D}\right]}=\left\langle\eta_{a}\left|L_{z, C}\right| \eta_{b}^{\left[q_{D}\right]}\right\rangle+\left\langle\eta_{a}\left|L_{z, C}^{\left[q_{D}\right]}\right| \eta_{b}\right\rangle+\left\langle\eta_{a}^{\left[q_{D}\right]}\left|L_{z, C}\right| \eta_{b}\right\rangle
$$

The first (last) term only contributes if $\eta_{b}\left(\eta_{a}\right)$ is centered on atom $D$. The middle term only contributes if $C \equiv D$. If all three contribute, the integral is zero by translational invariance.

If $\eta_{a}$ is centered on atom $D$, we use the properties of Gaussians (Eqn. 63) to write

$$
\begin{aligned}
\left\langle\eta_{a}^{\left[q_{D}\right]}\left|L_{z, C}\right| \eta_{b}\right\rangle & =-\left\langle\eta_{a}^{[q]}\left|L_{z, C}\right| \eta_{b}\right\rangle \\
& =-n_{a}^{q}\left\langle\eta_{a}^{q-}\left|L_{z, C}\right| \eta_{b}\right\rangle+2 \alpha_{a}\left\langle\eta_{a}^{q+}\left|L_{z, C}\right| \eta_{b}\right\rangle
\end{aligned}
$$


The same can be said of $\eta_{b}$.

To evaluate the $L_{z, D}^{\left[q_{D}\right]}$ term, we can use the translational invariance of the integral:

$$
\begin{aligned}
\left\langle\eta_{a}\left|L_{z, D}^{\left[q_{D}\right]}\right| \eta_{b}\right\rangle= & -\left\langle\eta_{a}^{\left[q_{A}\right]}\left|L_{z, D}\right| \eta_{b}\right\rangle-\left\langle\eta_{a}\left|L_{z, D}\right| \eta_{b}^{\left[q_{B}\right]}\right\rangle \\
= & \left\langle\eta_{a}^{[q]}\left|L_{z, D}\right| \eta_{b}\right\rangle+\left\langle\eta_{a}\left|L_{z, D}\right| \eta_{b}^{[q]}\right\rangle \\
= & n_{a}^{q}\left\langle\eta_{a}^{q-}\left|L_{z, C}\right| \eta_{b}\right\rangle-2 \alpha_{a}\left\langle\eta_{a}^{q+}\left|L_{z, C}\right| \eta_{b}\right\rangle \\
& +n_{a}^{q}\left\langle\eta_{a}\left|L_{z, C}\right| \eta_{b}^{q-}\right\rangle-2 \alpha_{a}\left\langle\eta_{a}\left|L_{z, C}\right| \eta_{b}^{q+}\right\rangle
\end{aligned}
$$

Thus, if one can evaluate the integrals with different values of angular momentum, one can also easily evaluate the gradient.

Let us consider two examples. The first example is when $C=D$ and $\eta_{a}$ and $\eta_{b}$ are not centered on atom $C$.

$$
\begin{aligned}
\left\langle\eta_{a}\left|L_{z, C}\right| \eta_{b}\right\rangle^{\left[q_{C}\right]} & =\left\langle\eta_{a}\left|L_{z, C}^{\left[q_{C}\right]}\right| \eta_{b}\right\rangle \\
& =\left\langle\eta_{a}^{[q]}\left|L_{z, C}\right| \eta_{b}\right\rangle+\left\langle\eta_{a}\left|L_{z, C}\right| \eta_{b}^{[q]}\right\rangle
\end{aligned}
$$

The second example is when $C=D$ and $\eta_{a}$ is centered on $C$, but $\eta_{b}$ is not.

$$
\begin{aligned}
\left\langle\eta_{c}\left|L_{z, C}\right| \eta_{b}\right\rangle^{\left[q_{C}\right]} & =\left\langle\eta_{c}^{\left[q_{C}\right]}\left|L_{z, C}\right| \eta_{b}\right\rangle+\left\langle\eta_{c}\left|L_{z, C}^{\left[q_{C}\right]}\right| \eta_{b}\right\rangle \\
& =-\left\langle\eta_{c}^{[q]}\left|L_{z, C}\right| \eta_{b}\right\rangle+\left(\left\langle\eta_{c}^{[q]}\left|L_{z, C}\right| \eta_{b}\right\rangle+\left\langle\eta_{c}\left|L_{z, C}\right| \eta_{b}^{[q]}\right\rangle\right) \\
& =\left\langle\eta_{a}\left|L_{z, C}\right| \eta_{b}^{[q]}\right\rangle
\end{aligned}
$$

[1] T. Itoh, Chemical Physics Letters 151, 166 (1988), ISSN 0009-2614.

[2] S. Sarkar, H. P. Hendrickson, D. Lee, F. DeVine, J. Jung, E. Geva, J. Kim, and B. D. Dunietz, Journal of Physical Chemistry C 121, 3771 (2017).

[3] N. A. Romero and D. A. Nicewicz, Chemical Reviews 116, 10075 (2016), pMID: 27285582.

[4] L. I. Schiff, Quantum Mechanics (McGraw-Hill, New Jersey, 1955).

[5] M. A. ElSayed, Journal of Chemical Physics 38, 2834 (1963).

[6] D. J. Griffiths, Introduction to Quantum Mechanics (Benjamin-Cummings Publishing Company, USA, 2004), 2nd ed.

[7] P. Abegg, Molecular Physics 30, 579 (1975), https://doi.org/10.1080/00268977500102151. 
[8] C. M. Marian, Wiley Interdisciplinary Reviews: Computational Molecular Science 2, 187 (2012), ISSN 1759-0884.

[9] J. C. Tully, Journal of Chemical Physics 93, 1061 (1990).

[10] B. F. E. Curchod, C. Rauer, P. Marquetand, L. Gonzlez, and T. J. Martnez, Journal of Chemical Physics 144, 101102 (2016).

[11] G. Granucci, M. Persico, and G. Spighi, Journal of Chemical Physics 137, 22A501 (2012).

[12] G. Cui and W. Thiel, The Journal of Chemical Physics 141, 124101 (2014), https://doi.org/10.1063/1.4894849.

[13] P. V. Parandekar and J. C. Tully, Journal of Chemical Physics 122, 094102 (2005).

[14] J. R. Schmidt, P. V. Parandekar, and J. C. Tully, Journal of Chemical Physics 129, 044104 (2008).

[15] M. F. Herman, The Journal of Chemical Physics 81, 754 (1984), https://doi.org/10.1063/1.447708.

[16] J. C. Arce and M. F. Herman, The Journal of Chemical Physics 101, 7520 (1994), https://doi.org/10.1063/1.468245.

[17] R. Kapral and G. Ciccotti, Journal of Chemical Physics 110, 8919 (1999).

[18] M. F. Herman, The Journal of Chemical Physics 103, 8081 (1995), https://doi.org/10.1063/1.470173.

[19] J. E. Subotnik, W. Ouyang, and B. R. Landry, Journal of Chemical Physics 139, 214107 (2013).

[20] G. A. Meek and B. G. Levine, Journal of Physical Chemistry Letters 5, 2351 (2014).

[21] S. Hammes-Schiffer and J. Tully, Journal of Chemical Physics 101, 4657 (1994).

$[22]$ T. Nelson, S. Fernandez-Alberti, A. E. Roitberg, and S. Tretiak, Chemical Physics Letters 590, 208 (2013).

[23] R. Kapral, Chemical Physics 481, 77 (2016), ISSN 0301-0104, quantum Dynamics and Femtosecond Spectroscopy dedicated to Prof. Vladimir Y. Chernyak on the occasion of his 60th birthday.

[24] We note that ISC multiple spawning dynamics [10, 59, 60] should be far less sensitive to the choice of adiabatic versus diabatic basis.

[25] Indeed, recent work with reasonably expensive methods (like EOM-CCSD and ADC(2)) has already begun calculating the effect of SOC on excited states 61 [4]. If these methods can 
handle SOC, certainly incorporating SOC should be feasible within a CIS energy calculation. The question remains, however, as to the feasibility of calculating a gradient.

[26] G. Granucci and M. Persico, Journal of Computational Chemistry 32, 2690 (2011), ISSN 1096-987X.

[27] We emphasize that we are not including the SOC operator when we optimize orbitals for the original HF calculation; as is well known, such an approach would be unbounded [65]. That being said, no such problem should arise in practice if SOC is included only within a post-HF CIS calculation.

[28] D. Maurice, Single electron theories of excited states (University of California, Berkeley, 1998).

[29] S. Fatehi, E. Alguire, Y. Shao, and J. E. Subotnik, Journal of Chemical Physics 135, 234105 (2011).

[30] Formally, $E_{A}^{[x]}$ is defined $E_{A}^{[x]} \equiv \sum_{\mathbf{a b i j}} X_{\mathbf{a i}}^{*} A_{\mathbf{i a j b}}^{[x]} X_{\mathbf{b j}}-\sum_{\mathbf{c k}} \Theta_{\mathbf{c k}}^{[x]} Y_{\mathbf{c k}}-E_{H F}^{[x]}$.

[31] Formally, $E_{A}^{[x]}$ is defined $E_{V}^{[x]} \equiv \sum_{\mathbf{a b i}} X_{\mathbf{a i}}^{*} V_{\mathbf{a b}}^{[x]} X_{\mathbf{b i}}-\sum_{\mathbf{a i j}} X_{\mathbf{a i}}^{*} V_{\mathbf{j i}}^{[x]} X_{\mathbf{a j}}+\sum_{\mathbf{c k}} \Theta_{\mathbf{c k}}^{[x]} Y_{\mathbf{c k}}^{S O C}$.

[32] J. Gerratt and I. M. Mills, The Journal of Chemical Physics 49, 1719 (1968), https://doi.org/10.1063/1.1670299.

[33] J. A. Pople, R. Krishnan, H. B. Schlegel, and J. S. Binkley, International Journal of Quantum Chemistry 16, 225 (1979).

[34] N. C. Handy and H. F. Schaefer, Journal of Chemical Physics 81, 5031 (1984).

[35] Y. Shao, Z. Gan, E. Epifanovsky, A. T. Gilbert, M. Wormit, J. Kussmann, A. W. Lange, A. Behn, J. Deng, X. Feng, et al., Molecular Physics 113, 184 (2015), https://doi.org/10.1080/00268976.2014.952696.

[36] E. R. Davidson, J. Comp. Phys. 17, 87 (1975).

[37] Y. Notay, SIAM Journal on Matrix Analysis and Applications 26, 522 (2004), https://doi.org/10.1137/S0895479803430941.

[38] S. Mai, T. Mller, F. Plasser, P. Marquetand, H. Lischka, and L. Gonzlez, The Journal of Chemical Physics 141, 074105 (2014).

[39] E. Epifanovsky, K. Klein, S. Stopkowicz, J. Gauss, and A. I. Krylov, The Journal of Chemical Physics 143, 064102 (2015).

[40] X. Gao, S. Bai, D. Fazzi, T. Niehaus, M. Barbatti, and W. Thiel, Journal of Chemical Theory and Computation 13, 515 (2017), pMID: 27959528, https://doi.org/10.1021/acs.jctc.6b00915.

[41] E. C. Alguire, S. Fatehi, Y. Shao, and J. E. Subotnik, Journal of Physical Chemistry A 118, 
11891 (2014).

[42] A. Savin, in Recent Advances in Density Funtional Methods, edited by D. P. Chong (World Scientific, 1995), pp. 129-154.

[43] A. Savin and H. J. Flad, International Journal of Quantum Chemistry 56, 327 (1995).

[44] P. M. W. Gill, R. D. Adamson, and J. A. Pople, Molecular Physics 88, 1005 (1996).

[45] Y. Tawada, T. Tsuneda, S. Y. T. Yanai, , and K. Hirao, Journal of Chemical Physics 120, 8425 (2004).

[46] J. W. Song, T. Hirosawa, T. Tsuneda, and K. Hirao, Journal of Chemical Physics 126, 154105 (2007).

[47] T. Yanai, D. P. Tew, and N. C. Handy, Chemical Physics Letters 393, 51 (2004).

[48] O. A. Vydrov, J. Heyd, A. V. Krukau, and G. E. Scuseria, Journal of Chemical Physics 125, 234109 (1006).

[49] T. M. Henderson, B. G. Janesko, and G. E. Scuseria, Journal of Chemical Physics 128, 194105 (2008).

[50] J. D. Chai and M. Head-Gordon, Journal of Chemical Physics 128, 084106 (2008).

[51] M. A. Rohrdanz and J. M. Herbert, Journal of Chemical Physics 129, 034107 (2008).

[52] M. A. Rohrdanz, K. M. Martins, and J. M. Herbert, Journal of Chemical Physics 130, 054112 (2009).

[53] A. W. Lange, M. A. Rohrdanz, and J. M. Herbert, Journal of Physical Chemistry B 112, 6304 (2008).

[54] R. Baer, E. Livshits, and U. Salzner, Annual Reviews in Physical Chemistry 61, 85 (2010).

[55] Q. Ou and J. E. Subotnik, Journal of Physical Chemistry C 117, 19839 (2013).

[56] M. Marazzi, S. Mai, D. Roca-Sanjun, M. G. Delcey, R. Lindh, L. Gonzlez, and A. Monari, Journal of Physical Chemistry Letters 7, 622 (2016).

[57] T. Mani and S. A. Vinogradov, The Journal of Physical Chemistry Letters 4, 2799 (2013), pMID: 24143268, https://doi.org/10.1021/jz401342b.

[58] H. F. King and T. R. Furlani, Journal of Computational Chemistry 9, 771 (1988), https://onlinelibrary.wiley.com/doi/pdf/10.1002/jcc.540090707.

[59] D. A. Fedorov, S. R. Pruitt, K. Keipert, M. S. Gordon, and S. A. Varganov, The Journal of Physical Chemistry A 120, 2911 (2016), pMID: 27064356, https://doi.org/10.1021/acs.jpca.6b01406. 
[60] D. A. Fedorov, A. O. Lykhin, and S. A. Varganov, The Journal of Physical Chemistry A 122, 3480 (2018), pMID: 29533626, https://doi.org/10.1021/acs.jpca.8b00883.

[61] E. Epifanovsky, K. Klein, S. Stopkowicz, J. Gauss, and A. I. Krylov, The Journal of Chemical Physics 143, 064102 (2015).

[62] Z. Cao, F. Wang, and M. Yang, The Journal of Chemical Physics 145, 154110 (2016).

[63] C. M. Krauter, B. Schimmelpfennig, M. Pernpointner, and A. Dreuw, Chemical Physics 482, 286 (2017), ISSN 0301-0104, electrons and nuclei in motion - correlation and dynamics in molecules (on the occasion of the 70th birthday of Lorenz S. Cederbaum).

[64] D. Bokhan, A. Perera, D. N. Trubnikov, and R. J. Bartlett, The Journal of Chemical Physics 147, 164118 (2017).

[65] B. A. Hess, C. M. Marian, and S. D. Peyerimhoff, in Modern Electronic Structure Theory, Vol. I, edited by D. Yarkony (World Scientific, NJ, 1995), pp. 152-278. 